\title{
Superdiffusive transport in laboratory and astrophysical plasmas
}

\author{
G. Zimbardo ${ }^{1, \dagger}$, E. Amato ${ }^{2}$, A. Bovet ${ }^{3}$, F. Effenberger ${ }^{4,5}$, A. Fasoli ${ }^{3}$, \\ H. Fichtner ${ }^{6}$, I. Furno ${ }^{3}$, K. Gustafson ${ }^{3}$, P. Ricci $^{3}$ and S. Perri ${ }^{1}$ \\ ${ }^{1}$ Department of Physics, University of Calabria, Ponte P. Bucci, Cubo 31C, I-87036 Rende, Italy \\ ${ }^{2}$ INAF, Osservatorio Astrofisico di Arcetri, Largo E. Fermi 5, I-50125 Firenze, Italy \\ ${ }^{3}$ Ecole Polytechnique Federale Lausanne, EPFL, Lausanne, CH-1015, Switzerland \\ ${ }^{4}$ Department of Physics and KIPAC, Stanford University, Stanford, CA 94305, USA \\ ${ }^{5}$ Department of Mathematics, University of Waikato, P.B. 3105, Hamilton, New Zealand \\ ${ }^{6}$ Institut für Theoretische Physik IV, Ruhr-Universität Bochum, Universitätsstrasse 150, \\ 44780 Bochum, Germany
}

(Received 4 May 2015; revised 11 September 2015; accepted 14 September 2015)

In the last few years it has been demonstrated, both by data analysis and by numerical simulations, that the transport of energetic particles in the presence of magnetic turbulence can be superdiffusive rather than normal diffusive (Gaussian). The term 'superdiffusive' refers to the mean square displacement of particle positions growing superlinearly with time, as compared to the normal linear growth. The so-called anomalous transport, which in general comprises both subdiffusion and superdiffusion, has gained growing attention during the last two decades in many fields including laboratory plasma physics, and recently in astrophysics and space physics. Here we show a number of examples, both from laboratory and from astrophysical plasmas, where superdiffusive transport has been identified, with a focus on what could be the main influence of superdiffusion on fundamental processes like diffusive shock acceleration and heliospheric energetic particle propagation. For laboratory plasmas, superdiffusion appears to be due to the presence of electrostatic turbulence which creates long-range correlations and convoluted structures in perpendicular transport: this corresponds to a similar phenomenon in the propagation of solar energetic particles (SEPs) which leads to SEP dropouts. For the propagation of energetic particles accelerated at interplanetary shocks in the solar wind, parallel superdiffusion seems to be prevailing; this is based on a pitch-angle scattering process different from that envisaged by quasi-linear theory, and this emphasizes the importance of nonlinear interactions and trapping effects. In the case of supernova remnant shocks, parallel superdiffusion is possible at quasi-parallel shocks, as occurring in the interplanetary space, and perpendicular superdiffusion is possible at quasi-perpendicular shocks, as corresponding to Richardson diffusion: therefore, cosmic ray acceleration at supernova remnant shocks should be formulated in terms of superdiffusion. The possible relations among anomalous transport in laboratory, heliospheric, and astrophysical plasmas will be indicated. 


\section{Introduction}

Unravelling the transport properties in plasmas, both for thermal particles and for suprathermal and energetic particles, is a fundamental issue in both laboratory and astrophysical plasmas. For laboratory plasmas, reaching the goal of controlled nuclear fusion by magnetic confinement requires us to understand and control plasma transport. For astrophysical and space plasmas, the transport properties determine for example the intensity of the fluxes of solar energetic particles (SEPs), which are one of the main concerns of space weather, as well as the efficiency of energetic particle acceleration due to Fermi processes, eventually resulting in various cosmic ray populations which are of great interest in astrophysics. Beside normal diffusion, the so-called anomalous, non-diffusive transport has gained growing attention during the last two decades in many fields including laboratory plasma physics, as well as space and astrophysical plasmas.

In the case of non-diffusive transport, the mean square displacement of the random walker grows nonlinearly in time,

$$
\left\langle\Delta x^{2}\right\rangle \propto t^{\alpha}
$$

with $\alpha \neq 1$. In particular, when $\alpha>1$ we have superdiffusion, and when $\alpha<1$ we have subdiffusion. Such non-diffusive transport has been found in a very wide range of natural systems, including protein transport in cellular membranes (Kusumi et al. 2005; Ritchie et al. 2005), albatross food search strategies (Klafter \& Sokolov 2005), and Lévy glasses (Burresi et al. 2012). The nonlinear growth of the mean square displacement is related to the presence of non-Markovian, long-memory properties in time, and/or to non-local, long-range correlation properties in space (Bouchaud \& Georges 1990; Metzler \& Klafter 2000, 2004; Zaslavsky 2002; del-Castillo-Negrete et al. 2004; Perrone et al. 2013). The long-range correlations and long-memory effects are frequently found to be associated with nonlinear maps (Geisel et al. 1985; Metzler \& Klafter 2000), turbulent transport in fluids and plasmas (Richardson 1926; Klafter et al. 1987; Shlesinger et al. 1987; Zimbardo et al. 2000a; Carreras et al. 2001; Zimbardo 2005; Zimbardo et al. 2010), and numerical simulation of energetic particle transport in astrophysical plasmas (Zimbardo et al. 2006; Pommois et al. 2007; Shalchi \& Kourakis 2007; Tautz 2010; Zimbardo et al. 2012). In laboratory plasmas, anomalous diffusion was reported, among others, by Carreras et al. (2001), Mier et al. (2008), Gustafson et al. (2012b), Gustafson \& Ricci (2012), and Bovet et al. $(2014 a)$, the latter work also giving experimental evidence of both subdiffusion and superdiffusion. Both in the laboratory and in astrophysical plasmas, transport is influenced by the magnetic field line random walk induced by low-frequency magnetic turbulence (e.g. Webb et al. 2006). Indeed, because of turbulence the field lines themselves are subject to a stochastic motion in the plane perpendicular to the average magnetic field. In the limit of small gyroradius, particles follow the magnetic field lines; field line random walks can correspond to normal diffusion, but, because of the scattering of particles parallel to the magnetic field, particles trace back the field lines. This can give rise to subdiffusion in the direction perpendicular to the magnetic field, a regime which is known as 'double diffusion' in laboratory plasmas (Rechester \& Rosenbluth 1978; Krommes et al. 1983) and 'compound diffusion' in astrophysical plasmas (e.g. Zimbardo 2005; Webb et al. 2006; Shalchi 2010). In astrophysics, the implications of subdiffusion on particle acceleration were considered by Duffy et al. (1995) and Kirk et al. (1996), and indications of electron superdiffusion was found by Ragot \& Kirk (1997); in space plasmas, observational 
evidence of superdiffusion of energetic particles accelerated at interplanetary shocks was found by Perri \& Zimbardo (2007, 2008, 2009a,b), and Sugiyama \& Shiota (2011), and indications of superdiffusion of solar flare energetic particles by Trotta \& Zimbardo (2011).

Among the other statistical tools (see Perrone et al. 2013 for an overview), superdiffusive transport can be described in terms of a Lévy random walk, that is, in terms of a probabilistic description where the probability $\Psi$ of a random walker making a free path of length $x$ (forward or backward) in a time $t$ is given by (Geisel et al. 1985; Klafter et al. 1987; Shlesinger et al. 1987)

$$
\Psi(x, t)=A|x|^{-\mu-1} \delta(|x|-v t), \quad|x|>\ell_{0},
$$

where $v>0$ is the speed of the particle, $A$ is a normalization constant, and $\ell_{0}$ is the shortest free path length for which the above power law probability applies, with $\Psi$ being a regular, non-singular function for $x<\ell_{0}$. This free path probability leads, for $1<\mu<2$, to superdiffusion with $\alpha=3-\mu$. In the Lévy walk description, it is essential to have a coupling between free path length and free path duration, as expressed by the delta function in (1.2), in order to ensure the constant velocity of the particle undergoing the Lévy walk. When such coupling is not present, a statistical process called Lévy flights is obtained (e.g. Metzler \& Klafter 2004). For Lévy flights the free path length and time are not related, so that free paths of very different velocities are possible. On the other hand, subdiffusion is related to the presence of dynamical traps, that is, to the case of a power law distribution of 'trapping' or 'waiting' times for the random walker (e.g. Klafter et al. 1987; Metzler \& Klafter 2000).

Anomalous diffusion can also be described by extending the normal diffusion equation to the case of fractional derivatives

$$
\frac{\partial^{\beta} n}{\partial t^{\beta}}=C \frac{\partial^{\mu} n}{\partial|x|^{\mu}},
$$

where the fractional derivatives are integro-differential operators (e.g. Chukbar 1995; Stern et al. 2014). In the above equation, $n$ is the particle number density, $C$ is a constant, $0<\beta<1$ and $\mu=2$ implies subdiffusion, while $\beta=1$ and $1<\mu<2$ implies superdiffusion; when fractional derivatives on both time and space are used, the anomalous regimes are characterized by $\alpha=2 \beta / \mu$ (see Zaslavsky 2002; Perrone et al. 2013; Bovet et al. 2014a; Stern et al. 2014, for more details). This relation is different from the one given above for Lévy walks, even for $\beta=1$, but it can be shown that the same relation is obtained when the finite extent of the integration domain over $x$ is taken into account (Zumofen \& Klafter 1993; Perri et al. 2015).

Here we show a number of experimental examples, both from laboratory and from astrophysical plasmas, where superdiffusive transport has been identified, with a focus on what could be the main influence of superdiffusion on plasma confinement in laboratory devices, on diffusive shock acceleration in astrophysics, as well as on solar and heliospheric energetic particle propagation. Further, we show how the application of superdiffusion to the acceleration of cosmic rays at supernova remnants (SNRs) helps to explain the observed radio synchrotron radiation spectra. We also briefly discuss some concepts and tools which can be used to attain an improved theoretical understanding of anomalous transport. The comparison of superdiffusive transport in different environments like laboratory plasmas, space plasmas, and astrophysical 
plasmas is important because this can help to understand the physical origin of superdiffusion: the latter lies in the interaction of particles with turbulence, and indeed numerical simulations have shown that many different transport regimes can be obtained depending on the turbulence level, the turbulence anisotropy, the Kubo number, and the particle energy (e.g. Pommois et al. 2007; Shalchi 2010; Zimbardo et al. 2012). The analysis of transport in different experimental systems can indicate which ingredients are indeed more effective in originating superdiffusion.

In $\S 2$ we describe non-diffusive transport in laboratory plasmas, with special attention to the TORPEX device; beside the experimental results, we discuss the possible links between the burstiness of the measured ion current and the irregular time structure of SEP observations in near-Earth space. In $\S 3$ we present the tools to obtain the experimental evidences of superdiffusion of energetic particles accelerated by interplanetary shocks, using both a probabilistic approach and a fractional advection-diffusion equation. In $\S 4$ we show for the first time how the application of superdiffusion to electron acceleration at SNR shocks can explain the observations of hard radio spectral indices for synchrotron radiation. In $\S 5$ we give the conclusions and discuss the future perspectives.

\section{Non-diffusive transport in laboratory plasmas}

Due to the importance of controlling transport in magnetically confined plasmas, many numerical and theoretical efforts have been done to understand the properties of plasma transport under different configurations and experimental setups (e.g. Dendy et al. 2007; Sanchez et al. 2008; Dewhurst et al. 2010). In recent years, suprathermal ion transport has been extensively studied on the Toroidal Plasma Experiment, TORPEX, an experimental device particularly suited to single out the influence of various parameters in determining the nature of transport.

TORPEX (Fasoli et al. 2013) is a simple magnetized torus (SMT) with a major radius $R=1 \mathrm{~m}$ and a minor radius $a=0.2 \mathrm{~m}$, in which helical, open magnetic field lines are created by combining a dominant toroidal magnetic field $B_{t} \simeq 75 \mathrm{mT}$ with a much weaker vertical magnetic field $B_{v} \simeq 2 \mathrm{mT}$. The SMT configurations are characterized by the number $N$ of toroidal turns performed by a field line before hitting the vessel. Plasmas of different gases can be produced and sustained by injecting microwaves at $2.45 \mathrm{GHz}$ in the electron cyclotron (EC) frequency range. Typical electron temperature and density are $T_{e} \simeq 1-6 \mathrm{eV}$ and $n_{e} \simeq 10^{15}-10^{16} \mathrm{~m}^{-3}$, while the ions are cold $\left(T_{i}<1 \mathrm{eV}\right)$. TORPEX allows for easy diagnostic access and well-characterized plasma scenarios, thus overcoming the difficulties in direct measurements of suprathermal ion transport. These are limited by the harsh plasma environment in fusion-grade devices and by the difficulty of performing measurements in distant astrophysical plasmas. In addition, detailed knowledge of the turbulence characteristics and of the background plasmas, necessary to realistically model the transport of suprathermal ions, has been achieved in recent years in TORPEX (Ricci et al. 2011; Gustafson et al. 2012a,b).

For the present experiments, plasmas are created by a small level of microwave power $(\approx 400 \mathrm{~W})$ on the high-field side of the TORPEX device and with $N \approx 2$. This scenario is characterized by the presence of ideal interchange modes (with a perpendicular wave number $k_{\Delta} \simeq 35 \mathrm{rad} \mathrm{m}^{-1}$ and a wave number parallel to the magnetic field, $k_{\|} \simeq 0$ ). These ideal interchange modes are driven by the magnetic curvature and the pressure gradient and intermittently generate field-aligned plasma structures termed 'blobs', which propagate radially outward (Theiler et al. 2009; Furno et al. 2011). 

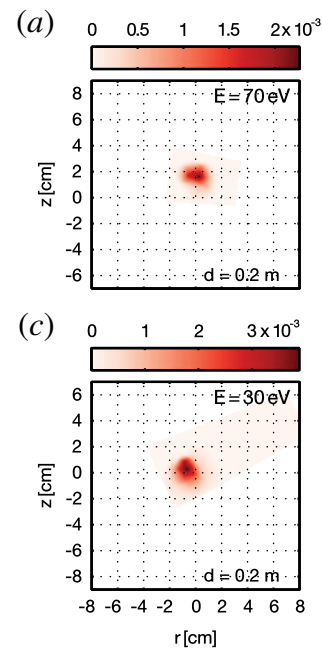

(b)

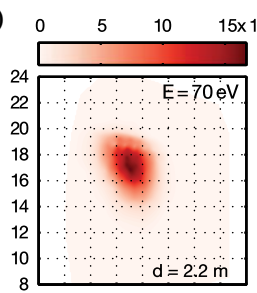

(d)

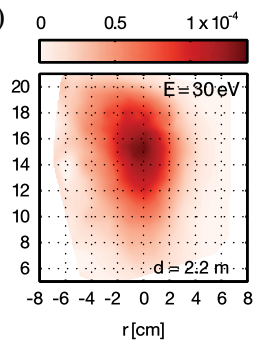

(e)

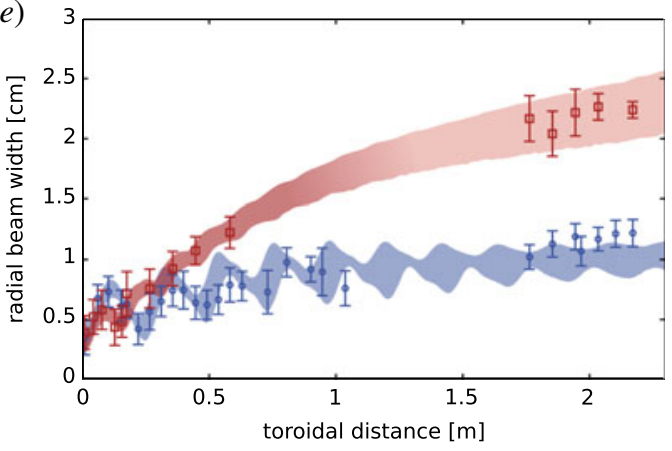

FIGURE 1. Poloidal suprathermal ion current profiles (units in $\mathrm{A} \mathrm{m}^{-2}$ ) at different toroidal distances for two ion energies $(a, b) E=70 \mathrm{eV},(c, d) E=30 \mathrm{eV}$. The vertical drift due to the curvature and gradient of the magnetic field is shown. The spreading due to the interaction with the turbulent plasmas is more important for $30 \mathrm{eV}$ ions. (e) Radial width of suprathermal ion current profiles as a function of toroidal distance. Red squares and blue circles represent experimental measurements for ions emitted at $30 \mathrm{eV}$ and $70 \mathrm{eV}$, respectively. Continuous bands are obtained from a synthetic diagnostic using numerical simulations for $30 \mathrm{eV}$ (red) and $70 \mathrm{eV}$ (blue) ions. The width of the bands is obtained by varying the simulation input parameters within experimental uncertainties. The different beam spreadings as a function of the toroidal distance indicate different transport regimes.

In these plasmas, suprathermal lithium $6^{+}$ions are injected in the blob region using a miniaturized ion source and detected using gridded energy analysers (GEAs), which were specifically developed for these experiments (Bovet et al. 2012, 2013, $2014 a, b)$. The suprathermal ion source is mounted on a moving system, which can continuously position the source at different toroidal locations. Two GEA detectors moving across almost the entire cross-section are installed at different toroidal distances along the suprathermal ion beam. This setup allows measurements of the three-dimensional properties of the suprathermal ion beam as it interacts with the plasma turbulence. For these measurements, two detection schemes can be used: (1) time-resolved measurements allowing for the evaluation of the statistical moments of the suprathermal ion current fluctuations and (2) synchronous detection to improve the signal to noise ratio, which allows for the measurement of the time-averaged profiles.

In figure 1, we show the spreading of the time-averaged suprathermal ion current profiles in the presence of plasma, measured at different toroidal locations for two different energies, $E=30 \mathrm{eV}$ (figure $1 a, b$ ) and $E=70 \mathrm{eV}$ (figure $1 c, d$ ), using the synchronous detection technique. The spreading of the suprathermal ion beam is quantified by the radial variance of the two-dimensional poloidal profile as a function of the toroidal distance, as shown in figure 1(e). In the present simple magnetized torus regime in the absence of a plasma, the motion of suprathermal ions corresponds to the gyromotion along the magnetic field lines at the cyclotron frequency, $f_{i} \simeq 188 \mathrm{kHz}$, which is superposed to an upward drift due to the curvature 
and gradient of the magnetic field. Figure 1 shows that on top of the unperturbed motion, a broadening due to the interaction with the plasma turbulence is present, which is different for the two suprathermal ion energies, hinting at different transport regimes. We note that, although the three-dimensional suprathermal ion beam profile is directly measured in TORPEX, in order to identify the transport regime the radial transport exponent, $\alpha_{R}$, should be computed from the temporal dependence of the variance of the displacement of the suprathermal ions. The experimental data of figure 1 are time averaged and the radial spreading of the ions is only accessible as a function of the toroidal distance.

To gain insight into the nature of transport, the experimental measurements are compared and analysed using results from numerical simulations with the help of a synthetic diagnostic reproducing the suprathermal ion current profile measured with the detectors. A large number of tracers $\left(1.6 \times 10^{5}\right)$ are injected in global fluid turbulent simulations that were previously validated against experimental data. The tracer trajectories are computed using the Newton equation of motion. Two simulations reproducing the experimental results are shown in figure 1(e) and are used to draw the bands in this figure, which quantify the simulation uncertainties.

Using the simulation results reproducing the experimental conditions, the value of the radial transport exponent, $\alpha_{R}$, can be readily computed from the evolution of the variance of the ion radial displacements as a function of time, $\sigma_{R}^{2}(t)$. At first, the transport of the ions is ballistic $\left(\sigma_{R}^{2}(t) \propto t^{2}\right)$, during a phase in which the ions have not yet interacted with the plasma and are not yet magnetized. The ions enter a second spreading phase as they start to interact with the plasma turbulence. In this phase, different transport regimes are observed, according to the energy of the ions and the character of the turbulence. A numerical study showed that in this interaction phase the transport can vary from a subdiffusive to a superdiffusive regime depending on two parameters that determine the relative sizes of the ion orbits and the turbulent structures: the injection energy normalized to the electron temperature, $E / T_{e}$, and the normalized fluctuations amplitude, e $\tilde{\phi} / T_{e}$ (Gustafson et al. 2012b). Here $\tilde{\phi}$ is the fluctuating electric potential due to the plasma turbulence. Fitting the temporal evolution of $\sigma_{R}^{2}$ to power laws provides the values of the transport exponents in the different phases.

In the interaction phase, an exponent $\alpha_{R}=0.51 \pm 0.01$ is found for ions of $70 \mathrm{eV}$ $\left(E / T_{e} \simeq 54\right)$ and $\alpha_{R}=1.20 \pm 0.04$ for ions of $30 \mathrm{eV}\left(E / T_{e} \simeq 23\right)$, indicating that the transport varies from subdiffusive to superdiffusive as the energy of the ions is decreased. For ions of $30 \mathrm{eV}$, after the superdiffusive phase, a phase where the transport is close to diffusive $\left(\alpha_{R}=0.92 \pm 0.04\right)$ is visible in figure $1(e)$ after approximately $1 \mathrm{~m}$. This phase appears when the size of the beam becomes sufficiently large that ions sample regions of the plasma with a different fluctuation amplitude, originating an average transport close to diffusive (Bovet et al. 2014a,b, 2015).

In order to relate the above results to theoretical studies, we can notice that there is a formal analogy between the transport of particles in the presence of electric fluctuations, if they obey the drift approximation, and the transport of magnetic field lines perpendicular to the magnetic field, in the case of two-dimensional fluctuations. Actually, in both cases a Hamiltonian structure for the field line equations or the particle motion equations can be found. In the former case, the electric drift velocity can be written as $\boldsymbol{v}_{E}=c \boldsymbol{E} \times \boldsymbol{B} / B^{2}$. Considering $x y$ as the plane perpendicular to $\boldsymbol{B}$, we have

$$
\frac{\mathrm{d} x}{\mathrm{~d} t}=-\frac{c}{B} \frac{\partial \tilde{\phi}}{\partial y}, \quad \frac{\mathrm{d} y}{\mathrm{~d} t}=\frac{c}{B} \frac{\partial \tilde{\phi}}{\partial x},
$$


where $x$ corresponds to the coordinate, $y$ to the impulse, and the Hamiltonian is given by $H=-c \tilde{\phi} / B$. Given that $\tilde{\phi}$ and $B$ depend only weakly on the coordinate along $\boldsymbol{B}$, in the limit of small gyroradius the particles follow the contours of $\tilde{\phi}$. In a similar way, for magnetic field line transport we have $\mathrm{d} x / \mathrm{d} z=B_{x} / B_{z}$ and $\mathrm{d} y / \mathrm{d} z=B_{y} / B_{z}$, which, in terms of the vector potential $\boldsymbol{A}(x, y)$, assuming two-dimensional magnetic fluctuations, yields

$$
\frac{\mathrm{d} x}{\mathrm{~d} z}=\frac{1}{B} \frac{\partial A_{z}}{\partial y}, \quad \frac{\mathrm{d} y}{\mathrm{~d} z}=-\frac{1}{B} \frac{\partial A_{z}}{\partial x},
$$

with $z$ playing the role of time and the Hamiltonian given by $H=A_{z} / B$. Hence, magnetic field lines follow the contours of the vector potential.

This allows for a possible interpretation of TORPEX experimental results in terms of previous numerical studies of field line random walk, with the transport in the radial direction corresponding to transport in the plane perpendicular to the magnetic field. Several numerical studies show that anomalous transport (both subdiffusion and superdiffusion) is found for a small Kubo number $R$. The Kubo number is given, for magnetic field lines, by the product of the turbulence level $\delta B / B_{0}$ times the ratio of parallel and perpendicular correlation lengths $\lambda_{\|} / \lambda_{\perp}$ (e.g. Isichenko 1992). For the $\boldsymbol{E} \times \boldsymbol{B}$ drift, the Kubo number is given by the drift speed $c \delta E / B_{0}$ times the ratio of the correlation time of $\delta E$ (with $\delta E$ the fluctuating electric field amplitude, which is related to the fluctuating electric potential by $\delta \boldsymbol{E}=-\nabla \tilde{\phi}$ ) over the perpendicular correlation length $\tau_{\text {corr }} / \lambda_{\perp}$. What is found is that for $R<1$ we have: (i) anomalous transport, both for field lines and for particles (Zimbardo et al. 2000b; Pommois et al. 2007); (ii) the structure of magnetic flux tubes becomes distorted but does not look Gaussian, rather it looks convoluted and fractal. When this structure is crossed by spacecraft (because of advection by the solar wind) the so-called solar energetic particle dropouts are observed: these are due to the spacecraft seeing both regions filled with SEPs, magnetically connected with the energetic particle source, and empty regions, which are not magnetically connected to the source; some events also show a fine structure of filled/empty regions (Giacalone et al. 2000; Mazur et al. 2000; Zimbardo et al. 2004; Trenchi et al. 2013). On the contrary, for $R \gtrsim 1$ we have mostly normal diffusion, a nearly Gaussian structure for the magnetic flux tubes (sometimes very dense), and the trajectory of a random walker has fractal dimension equal to 2 (for time going to infinity) (Isichenko 1992).

In addition, when superdiffusion is found, one can argue that the spatial structure of the random walker trajectory is fractal, and the fractal dimension can be related to the superdiffusion exponent (Bouchaud \& Georges 1990; Isichenko 1992). If superdiffusion is due to a Lévy process described by the free path probability in (1.2), it is found that the fractal dimension of the trajectory in a plane is given by $D_{F}=\mu$. If one takes a one-dimensional cut of the trajectory in the plane perpendicular to the magnetic field, its fractal dimension is $D_{F}^{\text {cut }}=D_{F}-1=\mu-1$. We can try to relate the fractal dimension of the distribution of superdiffusive ions to the observed superdiffusion exponent $\alpha_{R} \simeq 1.24$ (Bovet et al. 2014a), so that $D_{F}=\mu=2 / \alpha \simeq 1.61$. Crossing such a structure, as happens in TORPEX because of the transverse displacements of the plasma column due to turbulence, can give an intermittent signal as the one found for lithium ions in the case of energy equal to $30 \mathrm{eV}$ (Bovet et al. 2014b), with a predicted fractal dimension in time of order $D_{F}^{\text {cut }}=\mu-1 \simeq 0.6$.

In summary, the intermittency in the ion current which is found in TORPEX in the case of superdiffusion could be interpreted on the basis of the fact that for Kubo 
numbers of order one or less, anomalous transport perpendicular to the magnetic field is possible, and the corresponding structure of the magnetic flux tubes which are populated by lithium ions becomes convoluted and fractal, corresponding to the properties (i) and (ii) above. Indeed, the Kubo number for TORPEX experiments is estimated to be of order one. Therefore, this suggests that the anomalous behaviour is due to the structures induced by electric field turbulence, in agreement with the fact that superdiffusion and the intermittency of the current are not found for higher energy ions, which, having a larger gyroradius, are less influenced by the spatial structure of $\delta \boldsymbol{E}$.

\section{Superdiffusive transport at heliospheric shocks}

In numerical simulations of transport as well as in the particular case of lithium ions in the TORPEX device, one can trace the particle trajectories and hence directly obtain the mean square displacement and the corresponding transport properties. However, this is hardly possible in space plasmas, and certainly not in astrophysical plasmas. Therefore, for many systems the transport properties have to be obtained indirectly, considering the effects that transport has on some observable quantities. Space plasmas are in an intermediate situation, since a number of plasma properties can be measured in situ by spacecraft. In this context, Perri \& Zimbardo $(2007,2008)$ and Perri et al. (2015) have developed some diagnostic tools to extract the transport properties of energetic particles accelerated at heliospheric shocks by the measured intensity profiles of the energetic particles.

The method is based on the fact that in the case of superdiffusive transport described by a Lévy walk, the probability density function of positions, i.e. the propagator $P(x, t)$, is not a Gaussian but rather a probability distribution with power law tails, similar to a Lévy distribution. This can be obtained by solving the Montroll-Weiss equation in Fourier-Laplace space (e.g. Zumofen \& Klafter 1993): for small values of the scaling variable $\xi=\left(x / \ell_{0}\right) /\left(t / t_{0}\right)^{1 / \mu} \ll 1$ one has a modified Gaussian,

$$
P(x, t) \simeq \frac{\Gamma((\mu+1) / \mu)}{\pi(C t)^{1 / \mu}} \exp \left[-\frac{\Gamma(3 / \mu)}{2 \Gamma(1 / \mu)}\left[\frac{x}{(C t)^{1 / \mu}}\right]^{2}\right],
$$

where $\Gamma$ is the Euler gamma function and the constant $C$ is given by

$$
C=2 \frac{\mu-1}{\mu+1}\left|\cos \left(\frac{\pi}{2} \mu\right)\right| \Gamma(-\mu) \frac{\ell_{0}^{\mu}}{t_{0}}
$$

(Zumofen \& Klafter 1993; Zimbardo \& Perri 2013; Perri et al. 2015). In the definition of $\xi, \ell_{0}$ is the shortest length for which the free paths have a power law probability distribution, see (1.2), and $t_{0}=\ell_{0} / v$ is the corresponding time. Conversely, for large distances, $\xi \gg 1$ but $|x|<v t$, one obtains a power law propagator,

$$
P(x, t) \simeq \frac{\Gamma(\mu+1)}{\pi} \sin \left[\frac{\pi}{2} \mu\right]|C| \frac{t}{|x|^{\mu+1}},
$$

with the propagator going to zero for $x>v t$ (Blumen et al. 1990; Zumofen \& Klafter 1993). As can be seen, the long-distance propagator has a power law form, basically different from the normal Gaussian propagator. Other forms of non-Gaussian 
propagators in the astrophysical context have been discussed by Ragot \& Kirk (1997) and by Webb et al. (2006).

The propagator allows us to compute the density of particles of a given energy at any position in space and time as (e.g. Ragot \& Kirk 1997)

$$
n(x, t)=\int_{-\infty}^{\infty} \mathrm{d} x^{\prime} \int_{-\infty}^{t} \mathrm{~d} t^{\prime} P\left(x-x^{\prime}, t-t^{\prime}\right) Q_{s h}\left(x^{\prime}, t^{\prime}\right),
$$

where $Q_{s h}=\Phi_{0} \delta\left(x^{\prime}-V_{s h} t^{\prime}\right)$ indicates the particle source, located at the shock which is moving with speed $V_{s h}$. Here $\Phi_{0}$ is the flux of energetic particles, assumed to be injected at the shock. When using the propagator for Lévy walks, (3.4) allows us to obtain some of the most important modifications of the standard scenario due to superdiffusive transport. First, we can compute the particle density far upstream of the shock, where the form (3.3) of the propagator applies, which yields

$$
n(x, t) \simeq \frac{\Gamma(\mu+1)}{\pi} \frac{\sin (\pi \mu / 2)|C| \Phi_{0}}{\mu(\mu-1)} \frac{\left(x-V_{s h} t\right)^{1-\mu}}{V_{s h}^{2}}
$$

(Perri \& Zimbardo 2007, 2008). We can set the observer time at $t=0$ (like in a snapshot), so that far off the shock the spatial profile of the accelerated particles is a power law decay with slope $a=\mu-1$. This is markedly different from the predictions of normal diffusion, which yields an exponential profile upstream of the shock (e.g. Lee \& Fisk 1982). On the other hand, considering the varying level of Alfvén waves generated by cosmic rays streaming away from the shock, Bell (1978) obtained an energetic particle profile which is a power law with slope $a=1$. However, superdiffusive transport yields a power law profile even in the case of constant turbulence level, constant turbulence anisotropy (Perri et al. 2009; Perri \& Balogh 2010), and constant transport properties (Perri \& Zimbardo 2012b): accordingly, an energetic particle power law profile with $0<a<1$ implies superdiffusive transport with $\alpha=3-\mu=2-a$. In figure 2, we show an example of energetic particle fluxes measured by the Voyager 2 spacecraft at the termination shock, the first boundary of the heliosphere. The obtained values of the slope are $a=0.69-0.71$, so that superdiffusion with $\alpha \simeq 1.3$ is deduced for ions with energies from $200 \mathrm{keV}$ to 3.5 MeV (Perri \& Zimbardo 2009b).

It is interesting to note that similar results for the power law density profile upstream of the shock can be obtained using a fractional diffusion-advection equation of the form (e.g. Stern et al. 2014)

$$
\frac{\partial n}{\partial t}=\kappa \frac{\partial^{\mu} n}{\partial|x|^{\mu}}+u \frac{\partial n}{\partial x}+\delta(x)
$$

where $\kappa$ is the superdiffusion coefficient, with dimensions length ${ }^{\mu} /$ time, $1<\mu<2$ is the fractional exponent for superdiffusion, and $u$ is the background advection speed. The fractional spatial derivative is given by the Riesz derivative, defined as

$$
\frac{\partial^{\mu} n(x, t)}{\partial|x|^{\mu}}=\frac{1}{\pi} \sin \left(\frac{\pi}{2} \mu\right) \Gamma(1+\mu) \int_{0}^{\infty} \frac{n(x+\xi)-2 n(x)+n(x-\xi)}{\xi^{1+\mu}} \mathrm{d} \xi
$$

(Samko et al. 1993; Saichev \& Zaslavsky 1997). This can be regarded as a fractional generalization of the usual Laplace operator, so that (3.6) is the fractional analogue of the standard diffusion-advection equation. The latter is recovered in the limit $\mu=2$, 


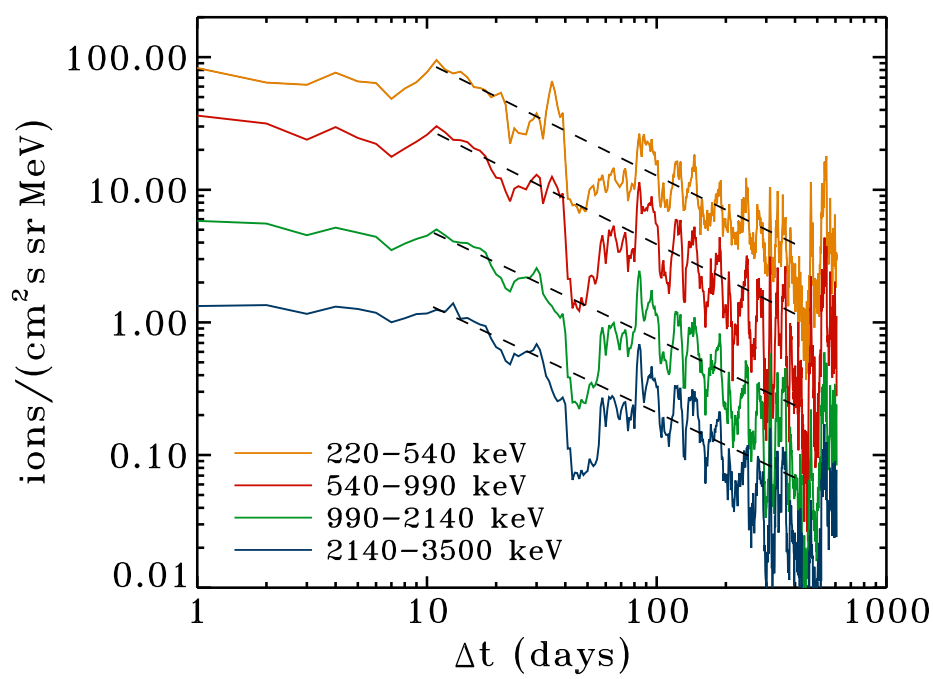

FIGURE 2. Energetic ion fluxes for several energy channels (as indicated) measured upstream of the termination shock, as a function of $\Delta t=\left|t-t_{\text {shock }}\right|$. The fits to the power law profiles are shown by dashed lines. Adapted from Perri \& Zimbardo (2012b).

and gives the above-mentioned well-known exponential upstream density profile for shock-injected particles. Asymptotic expressions for solutions to the fractional diffusion-advection equation are discussed in Litvinenko \& Effenberger (2014). We only give a short summary of these results here.

An important observation is that the asymptotic form of the propagator obtained from (3.6), without advection and a delta source in space and time, is identical to the leading order power law form of the Lévy walk propagator, i.e. (3.3) upon identifying the constant $|C|$ with $\kappa$. The solution to (3.6) is given by

$$
n(x, t)=\int_{0}^{t} P\left(x+u t^{\prime}, t^{\prime}\right) \mathrm{d} t^{\prime} .
$$

From this relation, two limiting cases for $n(x, t)$ with $x \gg u t$ and $0<x \ll u t$ can be derived. By changing the reference frame, the power law expression for the upstream particle density, as given by (3.5), can be recovered from these results in the limit of a very distant initial shock position. The actual approach of the solution to such a steady-state situation is an interesting field of study that is accessible now with a formulation in terms of a fractional transport equation. Litvinenko \& Effenberger (2014) verified their results with complementary solution methods using a weak diffusion approximation and a Fourier-series solution on a finite domain (Stern et al. 2014). Furthermore, the relation $\alpha=3-\mu$ is recovered for the time dependence of the variance of particle displacement when a finite particle speed is considered (see also the discussion in Perri et al. 2015).

An additional, complementary solution method for fractional diffusion equations is based on a generalization of the equivalence between stochastic differential equations (SDEs) and the Fokker-Planck equation (Gardiner 2009). The Wiener process representing the stochastic driver in the usual SDE formulation is generalized to Lévy motions $L_{\mu}(t)$ obeying the Fourier transform characteristic

$$
\mathcal{F}\left\{\mathrm{e}^{\mathrm{i} k L_{\mu}(t)}\right\}=\mathrm{e}^{-t|k|^{\mu}} .
$$


Identifying again $\kappa$ and $|C|$, the Fourier inversion of this expression yields the limiting forms of the propagator given in (3.1) and (3.3). The reader is referred to Magdziarz \& Weron (2007), Bovet et al. (2014a), and Effenberger (2014) for more details on solution methods and applications of this approach.

\section{Superdiffusive shock acceleration and hard electron spectra at SNR shocks}

An important implication of anomalous diffusion is that particle acceleration mechanisms based on diffusive, stochastic motion are modified because the underlying statistical process is not Gaussian. In particular, the cosmic ray energization mechanism is thought to be the diffusive shock acceleration (DSA), a first-order Fermi process which works both for ions and electrons (e.g. Bell 1978; Drury 1983; Perri et al. 2011; Balogh et al. 2013; Giacalone 2013; Amato 2014). It was shown by Duffy et al. (1995) and by Kirk et al. (1996) that in the case of subdiffusion the acceleration time and the energy spectral index are modified, and an application to the diffuse radio emission observed from the Coma cluster of galaxies was proposed (Ragot \& Kirk 1997). The extension to the superdiffusive case was carried out by Perri \& Zimbardo (2012a) and by Zimbardo \& Perri (2013), and similar modifications to DSA in the case of Richardson diffusion were recently considered by Lazarian \& Yan (2014) (see below). Here we propose for the first time how application of superdiffusion to electrons accelerated at supernova remnants (SNRs) can help to explain the observed radio synchrotron radiation spectra.

Supernova remnant shocks are the most popular candidate for the acceleration of galactic cosmic rays (CRs) (e.g. Helder et al. 2012; Blasi 2013; Amato 2014). This association has gained important observational support in recent years thanks to the data collected by X-ray telescopes such as Chandra and XMM-Newton and gamma-ray telescopes such as Fermi and AGILE, with the former instruments showing accelerated electrons with energies up to tens of $\mathrm{TeV}$ (Reynolds et al. 2012; Vink 2012) and the latter highlighting for the first time the presence of relativistic protons in SNRs (Abdo et al. 2010a,b; Tavani et al. 2010; Giuliani et al. 2011). However, a number of issues on the actual acceleration processes remain open.

Information on the electron acceleration can be obtained from synchrotron emission at shell-type SNRs (Green 2009; Morlino et al. 2010; Reynolds et al. 2012; Vink 2012). For these SNRs one can confidently assume that electron acceleration happens at the blast wave of the supernova ejecta. The synchrotron radiation of several SNRs is observed both in the X-rays and in the radio bands. The latter radiation is due to electrons with energies in the range between a few hundred $\mathrm{MeV}$ and a few $\mathrm{GeV}$, and it is characterized by a spectral density $S_{v}$ at frequency $v$ :

$$
S_{v} \propto v^{-\alpha_{v}}
$$

(Reynolds et al. 2012), where $\alpha_{v}$ is the power law spectral index in the radio band. This is related to the spectral index $\gamma$ of the electron differential energy distribution by (e.g. Longair 1994)

$$
\gamma=2 \alpha_{v}+1,
$$

so that observing the radio synchrotron emission gives information on the electron energy distribution. On the other hand, the well-known relation between the energy spectral index for ultrarelativistic particles and the shock compression ratio $r$ given by DSA is

$$
\gamma=\frac{r+2}{r-1},
$$




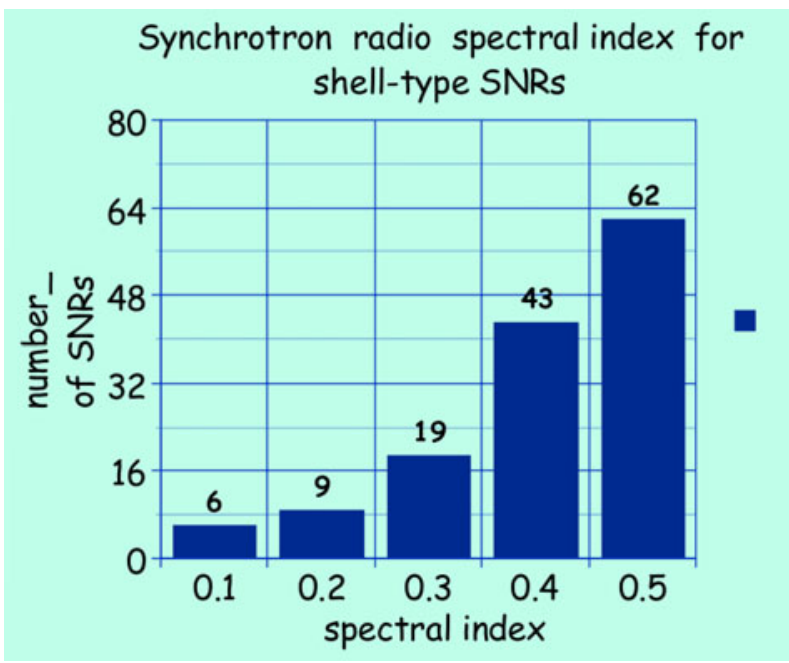

FIGURE 3. Distribution of synchrotron spectral indices $\alpha_{v}$ for shell-type SNRs. Adapted from Reynolds et al. (2012).

so that the compression ratio might be deduced from the observation of $\alpha_{v}$, if we restrict our reasoning to the test particle approach of DSA.

A puzzling property of the observed radio synchrotron spectral indices (Onić 2013), as obtained for instance from Green's catalogue (Green 2009), is that the distribution of $\alpha_{v}$ is peaked at 0.5 , implying $\gamma=2$ and $r=4$, but values of $\alpha_{v}$ substantially smaller than 0.5 are also found, see figure 3. These imply $\gamma<2$, which, according to (4.3), would require a compression ratio larger than 4 . However, for non-relativistic shock speeds, and if no distinction is made between the gas compression ratio and that due to the motion of the scattering centres (e.g. Vainio \& Schlickeiser 1999), $r=4$ is the largest compression ratio that can be obtained within the test-particle regime. We note that for the blast wave of SNRs, the shock speeds are in the range $10^{3}-10^{4} \mathrm{~km} \mathrm{~s}^{-1}$, and the sound speed in the interstellar medium is of the order of a few tens of $\mathrm{km} \mathrm{s}^{-1}$, so that the appropriate thermodynamical description is that of a strong non-relativistic shock, namely the Mach number $M a \gg 1$ and the compression ratio $r \approx 4$, unless efficient particle acceleration occurs.

In this latter case the total effective compression ratio can be increased by the fact that accelerated particles take away energy from the system, making the shock effectively radiative. Indeed, nonlinear shock acceleration theory, a framework which takes into account the backreaction of accelerated particles on the shock properties, predicts the formation of an extended cosmic ray precursor ahead of the fluid discontinuity, which now takes the name of subshock (e.g. Drury 1983; Amato \& Blasi 2005; Bykov et al. 2012; Reynolds et al. 2012): the overall compression ratio from far upstream to downstream becomes larger than what the Rankine-Hugoniot conditions would lead us to infer, while the compression at the fluid subshock becomes less than the expected value. Such a precursor is actually observed for the solar wind termination shock (TS) crossed by the Voyager 2 spacecraft (Decker et al. 2008; Richardson et al. 2008). Indeed, for the TS the energetic particle precursor allows us to go from the compression ratio of $r \simeq 2$ observed just at the shock (Decker et al. 2008; Richardson et al. 2008) to a global compression ratio of $r \simeq 2.4-2.8$ (Florinski et al. 2009; Arthur \& le Roux 2013). For SNRs, the extended precursor 
with increased compression ratio, perhaps as large as $r=7-8$, leads to a spectral hardening for higher energy particles which sample a larger region around the shock (Reynolds et al. 2012; Amato 2014). However, this is not the case for the $\mathrm{GeV}$ electrons emitting synchrotron radiation at radio wavelengths. Indeed, because of their limited energy and Larmor radius, they are expected not to diffuse far in the precursor, but rather stay close to the 'fluid subshock', and only experience the compression ratio there. Various models give a compression ratio at the subshock in the range $r=3-3.8$ (Reynolds 2008; Reynolds et al. 2012), corresponding to $\gamma=2.07-2.5$ and to $\alpha_{v}=0.53-0.74$. Therefore, the observed values of $\alpha_{v}>0.5$ can be explained by DSA with a compression ratio less than 4, but values of $\alpha_{v}<0.5$ (which are quite a few; see figure 3) cannot be interpreted within the same scheme. A somewhat similar problem is found for heliospheric shocks (Lee et al. 2012).

Here we propose that superdiffusive shock acceleration (SSA) (Perri \& Zimbardo 2012a) can explain those hard spectral indices. Indeed, a lower particle density far downstream leads to a modified probability of escape from the acceleration region in DSA (Kirk et al. 1996). A simple computation based on (3.4) shows that, instead of (4.3), SSA yields a new differential energy spectral index for shock accelerated particles,

$$
\gamma=\frac{6}{r-1} \frac{2-\alpha}{3-\alpha}+1,
$$

where $r$ is the shock compression ratio, $\alpha$ is the superdiffusion exponent, and where a corresponding expression can be obtained for non-relativistic particles (Perri \& Zimbardo 2012a; Zimbardo \& Perri 2013). We can say that the spectral index quantifies the statistical distribution of cosmic ray energies: since superdiffusion is due to a process, the Lévy walk, characterized by a statistics different from the Gaussian one, see (1.2), (3.1), and (3.3), the distribution of energies is also different, even if it is still a power law. Note that $\gamma$ depends both on the shock compression ratio and on the exponent of superdiffusion $\alpha$, so we can obtain a wide range of values for $\gamma$. In particular, for $\alpha>1$, harder spectral indices than those predicted by DSA are obtained. The above expression of $\gamma$ has been applied to the interpretation of energetic ion spectra at the termination shock by Perri \& Zimbardo (2012a) and to electron spectra at interplanetary shocks by Perri et al. (2015).

For SNRs, assuming that we can obtain $\gamma$ from the observed spectral index $\alpha_{\nu}$ for radio synchrotron emission, (4.2), and the compression ratio from modelling or other physical arguments, we can infer the superdiffusion exponent as

$$
\alpha=3 \frac{(\gamma-1)(r-1)-4}{(\gamma-1)(r-1)-6} .
$$

Assuming, as the most unfavourable case to superdiffusion, $r=4$, we obtain the values of the superdiffusion exponent plotted in figure 4 . We can see that hard electron spectra imply very strong superdiffusion with $\alpha$ up to 1.88 . It is interesting to notice that these values of $\alpha$ are consistent with those found for electrons in the heliosphere (Zimbardo et al. 2012). As a further example, let us consider young SNRs, for which $\alpha_{v} \simeq 0.6$ is typical. This can be explained by DSA with $r \leqslant 4$. However, many models predict a compression ratio $r \sim 3$ at the thermal subshock (Helder et al. 2012; Reynolds et al. 2012); this would give $\gamma \sim 2.5$ and $\alpha_{\nu} \sim 0.75$, which is larger than the observed value. Therefore, superdiffusion can allow us to explain the observations in this case too, SSA being able to yield harder spectral indices for a given compression ratio. 


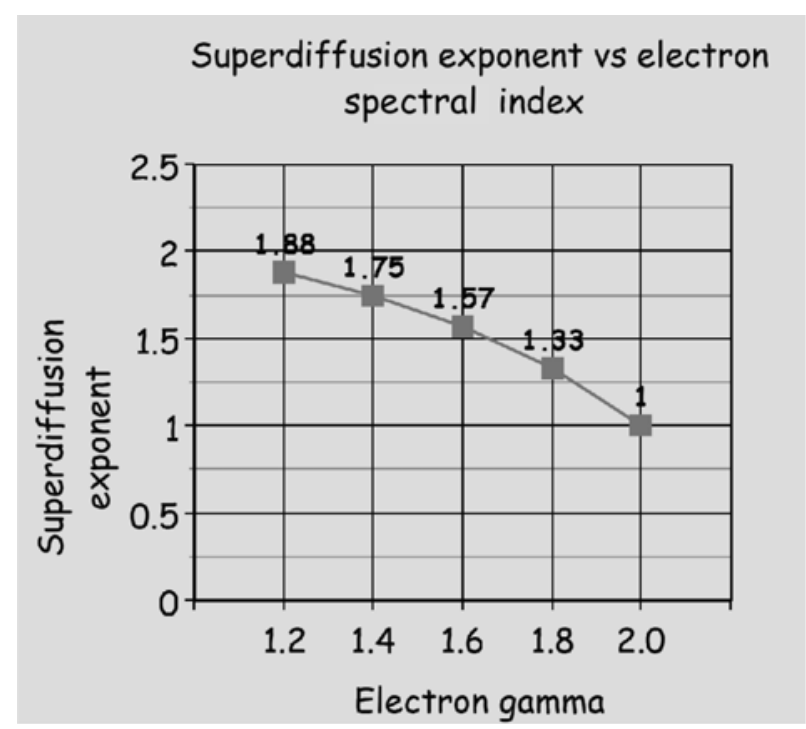

FIGURE 4. Superdiffusion exponent $\alpha$ versus the inferred electron spectral index $\gamma$, obtained from (4.5) for $r=4$. The exact values of $\alpha$ are indicated close to each data point.

The fact that the electron energy spectral index can be inferred from the synchrotron spectral index in the radio band, see (4.2), allows us to infer that superdiffusion can explain those observations. On the other hand, superdiffusion can be a very common process in astrophysics: recently Lazarian \& Yan (2014) emphasized the importance of Richardson superdiffusion in MHD turbulence for galactic cosmic rays. Richardson superdiffusion predicts that the spatial separation of magnetic field lines in the direction perpendicular to the mean magnetic field grows as $z^{3}$, with $z$ the coordinate along the average magnetic field. This superdiffusive regime holds up to field line separations comparable to the injection scale of turbulence, which in the galactic plane is of the order of 100 parsec, and hence much larger than the typical SNR size. This implies that both ions and electrons can undergo perpendicular superdiffusion; even in the case of parallel normal diffusion, $z \propto \sqrt{D_{\|} t}$, the perpendicular separation would grow as $\left\langle\Delta x^{2}\right\rangle \propto t^{1.5}$, following the same mechanism of compound diffusion (Webb et al. 2006). Clearly, the exponent of perpendicular superdiffusion can be larger in the case of parallel superdiffusion. Lazarian \& Yan (2014) also found that superdiffusion yields harder energy spectral indices than DSA (although they considered explicitly only the case $\alpha=1.5$ ), so that this can be a quite general property of cosmic rays accelerated in supernova environments dominated by MHD turbulence.

\section{Conclusions}

In this paper we have discussed the main statistical properties of anomalous diffusion, with an emphasis on superdiffusion. We have shown a number of experimental evidences of superdiffusion, both in laboratory plasmas and in astrophysical plasmas. For laboratory plasmas, superdiffusion appears to be due to the presence of electrostatic turbulence which creates long-range correlations and convoluted structures in perpendicular transport: on the one hand, this corresponds to a similar 
phenomenon in the propagation of SEPs which leads to SEP dropouts, as discussed in $\S 2$. On the other hand, superdiffusive perpendicular transport can also be interpreted in terms of Richardson diffusion for the separation of magnetic field lines, a phenomenon which is due to the multiscale nature of turbulence (Shlesinger et al. 1987), and which Lazarian \& Yan (2014) argued to hold in the galactic disc up to distances of the order of the turbulence injection scale at $\sim 100$ parsec. We can see that the long-range correlations induced by turbulence in plasmas can be effective over a very wide range of scales, from a few centimetres to a few tens of parsecs.

For the propagation of energetic particles accelerated at interplanetary shocks in the solar wind, parallel superdiffusion seems to be prevailing (e.g. Perri \& Zimbardo 2012b; Perri et al. 2015). On the other hand, for SNR shocks, superdiffusion can be needed to explain the observations of hard electron spectra; also, observations of several SNRs show that radio and X-ray emissions are not uniform around the SNR shell; rather, they are stronger at the opposite lobes, see, e.g., the images of SN1006 in Morlino et al. (2010). Those observations suggest that cosmic ray acceleration is strongly dependent on the angle between the interstellar magnetic field and the shock normal, but whether acceleration is more efficient at either quasi-parallel shocks or quasi-perpendicular shocks is not well known (see e.g. Orlando et al. 2007). In spite of the uncertainty of the influence of the magnetic field-shock normal angle, it is interesting to notice that superdiffusion is possible both at quasi-parallel shocks, as occurring in the interplanetary space, and at quasi-perpendicular shocks, as proposed by Lazarian \& Yan (2014). Therefore, cosmic ray acceleration at SNR shocks should be formulated in terms of superdiffusion for both cases.

On the other hand, the possibility of parallel superdiffusion is based on a pitch-angle scattering process different from that envisaged by quasi-linear theory (e.g. Drury 1983), where correlations between the pitch-angle changes are quickly lost. Conversely, numerical simulations show that anisotropic particle distribution functions are obtained also in the case of fully developed turbulence (Perrone et al. 2013): this points to the importance of nonlinear interactions and trapping effects (see also Shklyar \& Zimbardo 2014), which are outside the field of validity of quasi-linear theory.

We feel that a continued exchange of ideas and tools between laboratory and astrophysical plasmas can be very beneficial to the understanding of the transport properties and of their observable implications for both fields.

For future work, we can say that laboratory plasma experiments like TORPEX allow us to study with unprecedented detail the transport properties and the departures from Gaussian statistics of the tracer ions. The possibility of describing superdiffusion by means of fractional derivatives opens the gate to the formulation of superdiffusive shock acceleration in terms of a generalized, fractional Parker equation. The observations of hard electron spectral indices at SNR shocks can be explained reasonably well assuming that electron transport is superdiffusive and considering the extension of diffusive shock acceleration to the superdiffusive regime. Finally, it is interesting to notice that for some well-resolved SNR shocks it is possible to study the synchrotron emission profile upstream of the shock (e.g. Morlino et al. 2010). An analysis of the observations is under way, with the scope to single out the superdiffusive transport of electrons outside of the SNR blast wave.

\section{Acknowledgements}

The authors acknowledge support from the International Space Science Institute (ISSI) in Bern through the International Team no. 297 'Superdiffusive transport in space plasmas and its influence on energetic particle acceleration and propagation'. E.A. acknowledges support from the PRIN-INAF 2012 grant. 


\section{REFERENCES}

Abdo, A. A., Ackermann, M., Ajello, M., Baldini, L., Ballet, J., Barbiellini, G., Baring, M. G., Bastieri, D., Baughman, B. M., Bechtol, K. et al., Fermi Collaboration $2010 a$ Gamma-ray emission from the shell of supernova remnant W44 revealed by the Fermi LAT. Science 327, 1103.

Abdo, A. A., Ackermann, M., Ajello, M., Baldini, L., Ballet, J., Barbiellini, G., Bastieri, D., Baughman, B. M., Bechtol, K., Bellazzini, R. et al., Fermi COLlaboration $2010 \mathrm{~b}$ Observation of supernova remnant IC 443 with the Fermi large area telescope. Astrophys. J. 712, 459.

Amato, E. 2014 The origin of galactic cosmic rays. Intl J. Mod. Phys. D 23, 1430013.

AMATO, E. \& Blasi, P. 2005 A general solution to non-linear particle acceleration at non-relativistic shock waves. Mon. Not. R. Astron. Soc. Lett. 364, L76-L80.

Arthur, A. D. \& LE Roux, J. A. 2013 Particle acceleration at the heliospheric termination shock with a stochastic shock obliquity approach. Astrophys. J. Lett. 772, L26.

Balogh, A., Bykov, A., Lin, R. P., Raymond, J. \& Scholer, M. (Eds) 2013 Particle Acceleration in Cosmic Plasmas, Space Science Series of ISSI. Springer.

Bell, A. R. 1978 The acceleration of cosmic rays in shock fronts. I. Mon. Not. R. Astron. Soc. 182, 147.

BLASI, P. 2013 The origin of galactic cosmic rays. Astron. Astrophys. Rev. 21, 70.

Blumen, A., Klafter, J. \& Zumofen, G. 1990 A stochastic approach to enhanced diffusion: Lévy walks. Europhys. Lett. 13, 223-229.

Bouchaud, J. P. \& Georges, A. 1990 Anomalous diffusion in disordered media: statistical mechanisms, models and physical applications. Phys. Rep. 195, 127-293.

Bovet, A., FAsOli, A. \& Furno, I. $2014 a$ Time-resolved measurements of suprathermal ion transport induced by intermittent plasma blob filaments. Phys. Rev. Lett. 113, 225001.

Bovet, A., Fasoli, A., Ricci, P., Furno, I. \& Gustafson, K. 2015 Nondiffusive transport regimes for suprathermal ions in turbulent plasmas. Phys. Rev. E 91, 041101(R).

Bovet, A., Furno, I., Fasoli, A., Gustafson, K. \& Ricci, P. 2012 Investigation of fast ion transport in TORPEX. Nucl. Fusion 52, 094017.

Bovet, A., Furno, I., Fasoli, A., Gustafson, K. \& Ricci, P. 2013 Three-dimensional measurements of non-diffusive fast ion transport in TORPEX. Plasma Phys. Control. Fusion 55, 124021.

Bovet, A., Gamarino, M., Furno, I., Ricci, P., Fasoli, A., Gustafson, K., Newman, D. E. \& SÁNCHEZ, R. 2014b Transport equation describing fractional Lévy motion of suprathermal ions in TORPEX. Nucl. Fusion 54, 104009.

BURRESI, M. et al. 2012 Weak localization of light in superdiffusive random systems. Phys. Rev. Lett. 108, 110604.

Bykov, A. M., Ellison, D. C. \& Renaud, M. 2012 Magnetic fields in cosmic particle acceleration sources. Space Sci. Rev. 166, 71.

Carreras, B. A., Lynch, V. E. \& Zaslavsky, G. M. 2001 Anomalous diffusion and exit time distribution of particle tracers in plasma turbulence model. Phys. Plasmas 8, 5096.

Del-Castillo-Negrete, D., Carreras, B. A. \& Lynch, V. E. 2004 Fractional diffusion in plasma turbulence. Phys. Plasmas 11, 3854-3864.

Chukbar, K. V. 1995 Stochastic transport and fractional derivatives. Zh. Eksp. Teor. Fiz. 108, 1875.

Decker, R.-B., Krimigis, S. M., Roelof, E. C., Hill, M. E., Armstrong, T. P., Gloeckler, G., Hamilton, D. C. \& LAnzerotti, L. J. 2008 Mediation of the solar wind termination shock by non-thermal ions. Nature 454, 67-70.

Dendy, R. O., Chapman, S. C. \& PaCzuski, M. 2007 Fusion, space, and solar plasmas as complex systems. Plasma Phys. Control. Fusion 49, A95.

Dewhurst, J. M., Hnat, B. \& Dendy, R. O. 2010 Finite Larmor radius effects on test particle transport in drift wave-zonal flow turbulence. Plasma Phys. Control. Fusion 52, 025004.

DruRY, L. O'C. 1983 An introduction to the theory of diffusive shock acceleration of energetic particles in tenuous plasmas. Rep. Prog. Phys. 46, 973-1027. 
Duffy, P., Kirk, J.-G., Gallant, Y.-A. \& Dendy, R. O. 1995 Anomalous transport and particle acceleration at shocks. Astron. Astrophys. 302, L21.

EFFENBERGER, F. 2014 Energetic particle transport with stochastic differential equations: general methods and the extension to anomalous diffusion regimes. In 8th International Conference of Numerical Modeling of Space Plasma Flows (ASTRONUM 2013) (ed. N.i V. Pogorelov, E. Audit \& G. P. Zank), ASP Conference Series, vol. 488, p. 201. Astronomical Society of the Pacific.

Fasoli, A., Avino, F., Bovet, A., Furno, I., Gustafson, K., Jolliet, S., Loizu, J., Malinverni, D., RicCI, P., RivA, F. et al. 2013 Basic investigations of electrostatic turbulence and its interaction with plasma and suprathermal ions in a simple magnetized toroidal plasma. Nucl. Fusion 53, 063013.

Florinski, V., Decker, R. B., LE RouX, J. A. \& ZANK, G. P. 2009 An energetic-particle-mediated termination shock observed by Voyager 2. Geophys. Res. Lett. 36, L12101.

Furno, I., Spolaore, M., Theiler, C., Vianello, N., Cavazzana, R. \& Fasoli, A. 2011 Direct two-dimensional measurements of the field-aligned current associated with plasma blobs. Phys. Rev. Lett. 106, 245001.

Gardiner, C. W. 2009 Handbook of Stochastic Methods for Physics, Chemistry and the Natural Sciences. Springer.

Geisel, T., Nierwetberg, J. \& Zacherl, A. 1985 Accelerated diffusion in Josephson junctions and related chaotic systems. Phys. Rev. Lett. 54, 616-619.

Giacalone, J. 2013 Cosmic-ray transport and interaction with shocks. Space Sci. Rev. 176, 73.

Giacalone, J., JokipiI, R. \& MAzUR, J. E. 2000 Small-scale gradients and large-scale diffusion of charged particles in the heliospheric magnetic field. Astrophys. J. Lett. 532, L75-L78.

Giuliani, A., Cardillo, M., Tavani, M., Fukui, Y., Yoshimke, S., Torit, K., Dubner, G., Castelletti, G., Barbiellini, G., Bulgarelli, A. et al., AGile Collaboration 2011 Neutral pion emission from accelerated protons in the supernova remnant W44. Astrophys. $J$. Lett. 742, 30.

Green, D. A. 2009 A revised Galactic supernova remnant catalogue. Bull. Astron. Soc. India 37, 45.

Gustafson, K. \& RicCI, P. 2012 Lévy walk description of suprathermal ion transport. Phys. Plasmas 19, 032304.

Gustafson, K., Ricci, P., Bovet, A., Furno, I. \& Fasoli, A. $2012 a$ Suprathermal ion transport in simple magnetized torus configurations. Phys. Plasmas 19, 062306.

Gustafson, K., RicCi, P., Furno, I. \& FAsOli, A. $2012 b$ Nondiffusive suprathermal ion transport in simple magnetized toroidal plasmas. Phys. Rev. Lett. 108, 035006.

Helder, E. A., Vink, J., BYKov, A. M., Ohira, Y., RAYmond, J. C. \& Terrier, R. 2012 Observational signatures of particle acceleration in supernova remnants. Space Sci. Rev. 173, 369-431.

Isichenko, M. B. 1992 Percolation, statistical topography and transport in random media. Rev. Mod. Phys. 64, 961.

Kirk, J. G., Duffy, P. \& Gallant, Y. A. 1996 Stochastic particle acceleration at shocks in the presence of braided magnetic fields. Astron. Astrophys. 314, 1010.

Klafter, J., Blumen, A. \& Shlesinger, M. F. 1987 Stochastic pathway to anomalous diffusion. Phys. Rev. A 35, 3081-3085.

Klafter, J. \& Sokolov, I. M. 2005 Anomalous diffusion spreads its wings. Phys. World (August 2005), 29.

Krommes, J. A., Oberman, C. \& Kleva, R. B. 1983 Plasma transport in stochastic magnetic fields. Part 3. Kinetics of test particle diffusion. J. Plasma Phys. 30, 11.

Kusumi, A., Nakada, C., Ritchie, K., Murase, K., Suzuki, K., Murakoshi, H., Kasai, R. S., Kondo, J. \& Fujiwara, T. 2005 Paradigm shift of the plasma membrane concept from the two-dimensional continuum fluid to the partitioned fluid: high-speed single-molecule tracking of membrane molecules. Annu. Rev. Biophys. Biomol. Struct. 34, 351.

LAZARIAN, A. \& YAN, H. 2014 Superdiffusion of cosmic rays: implications for cosmic ray acceleration. Astrophys. J. 784, 38. 
LEE, M. A. \& FISK, L. A. 1982 Shock acceleration of energetic particles in the heliosphere. Space Sci. Rev. 32, 205.

Lee, M. A., Mewaldt, R. A. \& Giacalone, J. 2012 Shock acceleration of ions in the heliosphere. Space Sci. Rev. 173, 247.

Litvinenko, Y. E. \& EfFenberger, F. 2014 Analytical solutions of a fractional diffusion-advection equation for solar cosmic-ray transport. Astrophys. J. 796, 125.

LongaiR, M. S. 1994 High Energy Astrophysics, 2nd edn., vol. 2, pp. 229-251. Cambridge University Press.

Magdziarz, M. \& Weron, A. 2007 Competition between subdiffusion and Lévy flights: a Monte Carlo approach. Phys. Rev. E 75 (5), 056702.

Mazur, J. E., Mason, G. M., Dwyer, J. R., Giacalone, J., Jokipit, J. R. \& Stone, E. C. 2000 Interplanetary magnetic field line mixing deduced from impulsive solar flare particles. Astrophys. J. Lett. 532, L79-L82.

Metzler, R. \& Klafter, J. 2000 The random walk's guide to anomalous diffusion: a fractional dynamics approach. Phys. Rep. 339, 1-77.

Metzler, R. \& Klafter, J. 2004 Topical review: the restaurant at the end of the random walk: recent developments in the description of anomalous transport by fractional dynamics. J. Phys. A: Math. Gen. 37, R161-R208.

Mier, J. A., Sánchez, R., Garcia, L., Carreras, B. A. \& Newman, D. E. 2008 Characterization of nondiffusive transport in plasma turbulence via a novel Lagrangian method. Phys Rev. Lett. 101, 165001,1-4.

Morlino, G., Amato, E., Blasi, P. \& Caprioli, D. 2010 Spatial structure of X-ray filaments in SN 1006. Mon. Not. R. Astron. Soc. Lett. 405, L21-L25.

ONIĆ, D. 2013 On the supernova remnants with flat radio spectra. Astrophys. Space Sci. 346, 3-13.

Orlando, S., Bocchino, F., Reale, F., Peres, G. \& Petruk, O. 2007 On the origin of asymmetries in bilateral supernova remnants. Astron. Astrophys. 470, 927.

Perri, S. \& BAlogh, A. 2010 Stationarity in solar wind flows. Astrophys. J. 714, 937-943.

Perri, S., Yordanova, E., Carbone, V., Veltri, P., Sorriso-Valvo, L., Bruno, R. \& ANDRE, M. 2009 Magnetic turbulence in space plasmas: scale-dependent effects of anisotropy. J. Geophys. Res. 114, A02102.

Perri, S. \& Zimbardo, G. 2007 Evidence of superdiffusive transport of electrons accelerated at interplanetary shocks. Astrophys. J. Lett. 671, 177-180.

Perri, S. \& Zimbardo, G. 2008 Superdiffusive transport of electrons accelerated at corotating interaction regions. J. Geophys. Res. 113, A03107.

PERri, S. \& Zimbardo, G. $2009 a$ Ion and electron superdiffusive transport in the interplanetary space. Adv. Space Res. 44, 465-470.

PERRI, S. \& Zimbardo, G. $2009 b$ Ion superdiffusion at the solar wind termination shock. Astrophys. J. Lett. 693, L118-L121.

Perri, S. \& Zimbardo, G. $2012 a$ Superdiffusive shock acceleration. Astrophys. J. 750, 87.

Perri, S. \& Zimbardo, G. $2012 b$ Magnetic variances and pitch-angle scattering times upstream of interplanetary shocks. Astrophys. J. 754, 8.

Perri, S., Zimbardo, G., Effenberger, F. \& Fichtner, H. 2015 Parameter estimation of superdiffusive motion of energetic particles upstream of heliospheric shocks. Astron. Astrophys. 578, A2.

Perri, S., Zimbardo, G. \& Greco, A. 2011 On the energization of protons interacting with 3-D time-dependent electromagnetic fields in the Earth's magnetotail. J. Geophys. Res. 116, A05221.

Perrone, D., Dendy, R. O., Furno, I., Sanchez, R., Zimbardo, G., Bovet, A., Fasoli, A., Gustafson, K., Perri, S., Ricci, P. \& Valentini, F. 2013 Nonclassical transport and particle-field coupling: from laboratory plasma to the solar wind. Space Sci. Rev. 178, 233-270.

Pommois, P., Zimbardo, G. \& Veltri, P. 2007 Anomalous, non-Gaussian transport of charged particles in anisotropic magnetic turbulence. Phys. Plasmas 14, 012311,1-11. 
RaGot, B. R. \& Kirk, J. G. 1997 Anomalous transport of cosmic ray electrons. Astron. Astrophys. 327, 432.

Rechester, A. B. \& Rosenbluth, M. N. 1978 Electron heat transport in a Tokamak with destroyed magnetic surfaces. Phys. Rev. Lett. 40, 38-41.

Reynolds, S. P. 2008 Supernova remnants at high energy. Annu. Rev. Astron. Astrophys. 46, 89.

Reynolds, S. P., Gaensler, B. M. \& Bocchino, F. 2012 Magnetic fields in supernova remnants and pulsar-wind nebulae. Space Sci. Rev. 166, 231-261.

Ricci, P., Theiler, C., Fasoli, A., Furno, I., Gustafson, K., Iraji, D. \& Loizu, J. 2011 Methodology for turbulence code validation: quantification of simulation-experiment agreement and application to the TORPEX experiment. Phys. Plasmas 18, 032109.

Richardson, L. F. 1926 Atmospheric diffusion shown on a distance-neighbour graph. Proc. R. Soc. Lond. A 110, 709.

Richardson, J., Kasper, J. C., Wang, C., Belcher, J. W. \& Lazarus, A. J. 2008 Cool heliosheath plasma and deceleration of the upstream solar wind at the termination shock. Nature 454, 63.

Ritchie, K., Shan, X. Y., Kondo, J., Iwasawa, K., Fujiwara, T. \& Kusumi, A. 2005 Detection of non-Brownian diffusion in the cell membrane in single molecule tracking. Biophys. J. 88, $2266-2277$.

SaicheV, A. I. \& Zaslavsky, G. M. 1997 Fractional kinetic equations: solutions and applications. Chaos 7, 753-764.

Samko, S. S. G., Kilbas, A. A. A. \& Marichev, O. O. I. 1993 Fractional Integrals and Derivatives: Theory and Applications. Gordon and Breach.

Sanchez, R., Newman, D. E., Leboeuf, J.-N., DecyK, V. K. \& Carreras, B. A. 2008 Nature of transport across sheared zonal flows in electrostatic ion-temperature-gradient gyrokinetic plasma turbulence. Phys. Rev. Lett. 101, 205002.

SHALCHI, A. A. 2010 Unified particle diffusion theory for cross-field scattering: subdiffusion, recovery of diffusion, and diffusion in three-dimensional turbulence. Astrophys. J. 720, L127-L130.

Shalchi, A. \& Kourakis, I. 2007 A new theory for perpendicular transport of cosmic rays. Astron. Astrophys. 470, 405-409.

ShKlyar, D. R. \& Zimbardo, G. 2014 Particle dynamics in the field of two waves in a magnetoplasma. Plasma Phys. Control. Fusion 56, 095002.

Shlesinger, M. F., West, B. J. \& KlAfTER, J. 1987 Levy dynamics of enhanced diffusion: application to turbulence. Phys. Rev. Lett. 58, 1100.

Stern, R., EfFenberger, F., Fichtner, H. \& SChäFer, T. 2014 The space-fractional diffusionadvection equation: analytical solutions and critical assessment of numerical solutions. Fract. Calc. Appl. Anal. 17 (1), 171-190.

SugiYama, T. \& ShiotA, D. 2011 Sign for super-diffusive transport of energetic ions associated with a coronal-mass-ejection-driven interplanetary shock. Astrophys. J. 731, L34-L37.

TAUTZ, R. C. 2010 Simulation results on the influence of magneto-hydrodynamic waves on cosmic ray particles. Plasma Phys. Control. Fusion 52, 045016.

Tavani, M., Giuliani, A., Chen, A. W., Argan, A., Barbiellini, G., Bulgarelli, A., Caraveo, P., Cattaneo, P. W., Cocco, V., Contessi, T. et al., Agile Collaboration 2010 Direct evidence for hadronic cosmic-ray acceleration in the supernova remnant IC 443. Astrophys. J. Lett. 710, 151.

Theiler, C., Furno, I., Ricci, P., Fasoli, A., Labit, B., Muller, S. \& Plyushchev, G. 2009 Cross-field motion of plasma blobs in an open magnetic field line configuration. Phys. Rev. Lett. 103, 65001.

Trenchi, L., Bruno, R., Telloni, D., D’Amicis, R., Marcucci, M. F., Zurbuchen, T. H. \& WEBERG, M. 2013 Solar energetic particle modulations associated with coherent magnetic structures. Astrophys. J. 770, 11.

Trotta, E. M. \& Zimbardo, G. 2011 Quasi-ballistic and superdiffusive transport for impulsive solar particle events. Astron. Astrophys. 530, A130.

VAinio, R. \& SChlickeiser, R. 1999 Self-consistent Alfvén-wave transmission and test-particle acceleration at parallel shocks. Astron. Astrophys. 343, 303-311. 
VINK, J. 2012 Supernova remnants: the X-ray perspective. Astron. Astrophys. Rev. 20, 49.

Webb, G. M., Zank, G. P., Kaghashvili, E. Kh. \& Le RouX, J. A. 2006 Compound and perpendicular diffusion of cosmic rays and random walk of the field lines. I. Parallel particle transport models. Astrophys. J. 651, 211.

Zaslavsky, G. M. 2002 Chaos, fractional kinetics, and anomalous transport. Phys. Rep. 371, 461-580.

Zimbardo, G. 2005 Anomalous particle diffusion and Lévy random walk of magnetic field lines in three-dimensional solar wind turbulence. Plasma Phys. Control. Fusion 47, B755-B767.

Zimbardo, G., Greco, A., Sorriso-Valvo, L., Perri, S., Vörös, Z., Aburjania, G., Chargazia, K. \& AleXandrova, O. 2010 Magnetic turbulence in the geospace environment. Space Sci. Rev. 156, 89.

Zimbardo, G., Greco, A. \& Veltri, P. 2000a Superballistic transport in tearing driven magnetic turbulence. Phys. Plasmas 7, 1071-1074.

Zimbardo, G. \& PerRi, S. 2013 From Lévy walks to superdiffusive shock acceleration. Astrophys. J. 778, 35 .

Zimbardo, G., Perri, S., Pommois, P. \& Veltri, P. 2012 Anomalous particle transport in the heliosphere. Adv. Space Res. 49, 1633.

Zimbardo, G., Pommois, P. \& Veltri, P. 2004 Magnetic flux tube evolution in solar wind anisotropic magnetic turbulence. J. Geophys. Res. 109, A02113.

Zimbardo, G., Pommois, P. \& Veltri, P. 2006 Superdiffusive and subdiffusive transport of energetic particles in solar wind anisotropic magnetic turbulence. Astrophys. J. Lett. 639, L91-L94.

Zimbardo, G., Veltri, P. \& Pommois, P. $2000 b$ Anomalous, quasilinear, and percolative regimes for magnetic-field-line transport in axially symmetric turbulence. Phys. Rev. E 61, 1940-1948.

Zumofen, G. \& Klafter, J. 1993 Scale-invariant motion in intermittent chaotic systems. Phys. Rev. E 47, 851-863. 